\title{
Propionic acidemia: an extremely rare cause of hemophagocytic lymphohistiocytosis in an infant
}

\author{
Sultan Aydin Köker ${ }^{a}, M D$, Osman Yeşilbaş ${ }^{b}, M D$, Alper Kökerc, MD, and Esra Şevketoğlu ${ }^{d}$, Assoc. Prof.
}

\begin{abstract}
Hemophagocyticlymphohystiocytosis (HLH) may be primary (inherited/familial) or secondary to infections, malignancies, rheumatologic disorders, immune deficiency syndromes and metabolic diseases. Cases including lysinuric protein intolerance, multiple sulfatase deficiency, galactosemia, Gaucher disease, Pearson syndrome, and galactosialidosis have previously been reported. It is unclear how the metabolites trigger $\mathrm{HLH}$ in metabolic diseases. A 2-month-old infant with lethargy, pallor, poor feeding, hepatosplenomegaly, fever and pancytopenia, was diagnosed with HLH and the HLH-2004 treatment protocol was initiated. Analysis for primary HLH gene mutations and metabolic screening tests were performed together; primary HLH gene mutations were negative, but hyperammonemia and elevated methyl citrate were detected. Propionic acidemia was diagnosed with tandem mass spectrometry in neonatal dried blood spot. We report this case of HLH secondary to propionic acidemia. Both metabolic disorder screening tests and gene mutation analysis may be performed simultaneously especially for early diagnosis in infants presenting with HLH.

Key words: inborn errors metabolism, hemophagocytic lymphohistiocytosis, propionic academia.
\end{abstract}

http:/ / dx.doi.org/10.5546/aap.2020.eng.e174

To cite: Aydin Köker S, Yeşilbaş O, Köker A, Şevketoğlud E. Propionic acidemia: an extremely rare cause of hemophagocytic lymphohistiocytosis in an infant. Arch Argent Pediatr 2020; 118(2):e174-e177.

a. Department of Pediatric Hematology and Oncology.

b. Pediatric Intensive Care Unit.

University of Health Sciences, Van Training

and Research Hospital.

c. Hatay State Hospital, Pediatric Intensive Care Unit, Hatay.

d. University of Health Sciences, Bakirkoy Dr. Sadi Konuk Training and Research Hospital, Pediatric Intensive Care Unit, Istanbul.

Turkey.

E-mail address:

Sultan Aydin Koker, M.D.: drsultanaydin@hotmail.com

Funding: None.

Conflict of interest: None.

Received: 1-23-2019

Accepted: 9-9-2019

\section{INTRODUCTION}

Hemophagocytic lymphohistiocytosis $(\mathrm{HLH})$ is a life-threatening disorder in which there is uncontrolled proliferation of activated lymphocytes and histiocytes. The diagnosis of HLH is based on fulfilling at least five of eight criteria (fever, splenomegaly, bicytopenia, hypertriglyceridemia and/ or hypofibrinogenemia, hemophagocytosis, low/absent natural killer cell activity, hyperferritinemia, and high soluble interleukin2-receptor levels). HLH includes both familial and reactive disease triggered by infection, immunologic disorder, malignancy, or drugs. Clinical presentations of patients with primary (familial) and secondary (reactive) HLH are similar. ${ }^{1,2}$

The pathophysiology of HLH secondary to metabolic diseases is not clear. Childhood metabolic diseases such as lysinuric protein intolerance, methylmalonic acidemia, propionic acidemia (PA), galactosemia, Gaucher disease, multiple sulfatase deficiencies, Pearson syndrome, galactosialidosis, and biotinidase deficiency were noted to be associated with HLH. ${ }^{3,4}$

Both metabolic disease screening tests and gene mutation analysis performed simultaneously, especially in infants presenting with HLH, are important for early diagnosis of metabolic diseases. We want to report a rare case of HLH secondary to metabolic disorder associated with PA. In our case, PA was identified early due to performing metabolic disease tests and gene mutation analysis together.

\section{CASE PRESENTATION}

A two-month-old male infant from a first cousin marriage was referred to our pediatric intensive care unit with a three day history of lethargy, fever, pallor, poor feeding, and distended abdomen. He was born at 38 weeks gestation weighing $3470 \mathrm{~g}$. He had no significant family history. His physical examination was as follows: lethargic (Glasgow Coma Scale:11), mildly hypotonic, the pupils were miotic and reactive to light. He was tachycardic (182 beats/minute), normotensive $(74 / 42 \mathrm{mmHg})$, capillary refill time 
was 4 seconds (normal:1-2 second), respiratory rate $48 / \mathrm{min}$, and the temperature was $38.5^{\circ} \mathrm{C}$. Oxygen saturation was $94 \%$ in room air. The liver was palpable $5 \mathrm{~cm}$ and the spleen was palpable 2 $\mathrm{cm}$ below the costal margin. Laboratory findings were: $\mathrm{pH}$ : 7.12, $\mathrm{pCO}_{2} 17.8 \mathrm{mmHg}$, bicarbonate $11.9 \mathrm{mmol} / \mathrm{L}$, and lactate $4.2 \mathrm{mmol} / \mathrm{L}$ (normal range $0.4-2.2 \mathrm{mmol} / \mathrm{L}$ ). A complete blood count with differentials revealed a WBC level of $2200 / \mathrm{mm}^{3}$, a hemoglobin level of $7.2 \mathrm{~g} / \mathrm{dL}$, a hematocrit level of $20.4 \%$, and a platelet level of $24,000 / \mathrm{mm}^{3}$. Prothrombin time was 15.6 seconds, activated partial thromboplastin time was 27.1 seconds, fibrinogen level was $75 \mathrm{mg} / \mathrm{dL}$ (normal range 180-400 $\mathrm{mg} / \mathrm{dL}$ ), and $\mathrm{D}$-dimer was $400 \mathrm{ng} / \mathrm{mL}$ (normal range $0-240 \mathrm{ng} / \mathrm{mL}$ ). The other tests results were as follows: serum glucose $85 \mathrm{mg} / \mathrm{dL}$, urea $20.4 \mathrm{mg} / \mathrm{dL}$, creatinine $0.48 \mathrm{mg} / \mathrm{dL}$, uric acid $2.2 \mathrm{mg} / \mathrm{dL}$, albumin $3.02 \mathrm{mg} / \mathrm{dL}$, aspartate aminotransferase (AST) 17.3 U/L, alanine aminotransferase (ALT) $10.1 \mathrm{IU} / \mathrm{L}$, triglyceride $100.5 \mathrm{mg} / \mathrm{dL}$ (normal range $100-200 \mathrm{mg} / \mathrm{dL}$ ), lactate dehydrogenase (LDH) 292 U/L (normal range 120-300 U/L), ferritin $2,800 \mathrm{ng} / \mathrm{mL}$ (normal range 30-400 $\mathrm{ng} / \mathrm{mL}$ ), and ammonia $316 \mathrm{ug} / \mathrm{dL}$ (normal range 27-102 ug/dL). Serum electrolytes, C-reactive protein (CRP), procalcitonin, vitamin B12, immunoglobulin (Ig G, IgA, IgM, and IgE) were within normal limits.

The patient was intubated for invasive mechanical ventilation support, and sedationanalgesic medications, broad spectrum antibiotics, fluid resuscitation $(60 \mathrm{~mL} / \mathrm{kg}$ of $0.9 \%$ sodium chloride), maintenance intravenous fluid, and inotrope (adrenaline) were begun due to circulatory failure and preliminary diagnosis of septic shock. Bone marrow aspiration was performed due to the presence of fever, pancytopenia, hypofibrinogenemia, hyperferritinemia, and hepatosplenomegaly. While no storage cells were detected, hemophagocytosis was observed in bone marrow Wright staining. Repeated blood, tracheal aspirate, and urine cultures were sterile. Serological tests for viruses and bacteria (measles, rubella, mumps, Ebstein-Barr virus, cytomegalovirus, human immunodeficiency virus, hepatitis A-B-C, Brucella, and Salmonella) were negative. Hemophagocytic lymphohistiocytosis was diagnosed owing to the findings of fever, pancytopenia, splenomegaly, hypofibrinogenemia, hyperferritinemia, and hemophagocytosis in bone marrow aspirate. Natural killer cell activity and sCD25 were not studied because of technical impossibility. No pathologic findings were detected on brain magnetic resonance imaging. Both primary HLH gene mutation analyses and metabolic disease screening tests were performed simultaneously. While primary HLH gene mutation analyses such as perforin (PRF1), syntaxin-11 (STX11), and Munc13-4 (UNC13D) were negative, metabolic disease screening test results were compatible with PA (elevated propionylcarnitine level [8.92 $\mu \mathrm{M}$, cutoff $<6 \mu \mathrm{M}$ ], elevated urinary 3-hydroxy- propionic acid [153, cutoff: $<1.1]$ and methylcitric acid [34.0 $\mu \mathrm{M}$, cutoff: $<1.0 \mu \mathrm{M}]$ ). Intravenous immunoglobulin $(1 \mathrm{~g} / \mathrm{kg})$, the HLH2004 treatment protocol ${ }^{6}$ (dexamethasone, cyclosporine, and etoposide), high caloric nutrition, protein restricted diet, supplementation of daily L-carnitine, biotin, and coenzyme $Q$ were initiated. Continuous venovenous hemodialysis (CVVHD) was performed (thedialysate flow rate was set at $4000 \mathrm{~mL} / \mathrm{h} / 1.73 \mathrm{~m}^{2}$ ) due to hyperammonemia $(627 \mathrm{ug} / \mathrm{dL})$ which was unresponsive to medical therapies.

On the seventh day of PA and HLH-2004 treatment protocol, ${ }^{5}$ pancytopenia was improved, the ferritin level decreased below $500 \mathrm{ng} / \mathrm{mL}$, and the fibrinogen level increased to $167 \mathrm{mg} / \mathrm{dL}$. The dialysate flow rate was gradually decreased and CVVHD was terminated after the ammonia level decreased to normal values. On the $15^{\text {th }}$ day of the treatments, the patient's general condition was improved and he was extubated. All clinical and laboratory findings had completely resolved with the exception of hepatomegaly. The HLH-2004 chemotherapy protocol ${ }^{5}$ was discontinued after 8 weeks. The drugs related to PA were begun by the department of metabolic diseases. No HLH activation or metabolic crisis were observed during the 2 month follow-up period in the Departments of Metabolic Disease and Pediatric Hematology and Oncology.

\section{DISCUSSION}

Hemophagocytic lymphohistiocytosis most frequently affects infants from birth to 18 months of age, but the disease may also be observed in children and adults of all ages. ${ }^{1}$ Primary (familial) HLH caused by gene mutations include FHL1, FHL2(PRF1/Perforin), FHL3 (UNC13D/ Munc13-4), FHL4 (STX11/Syntaxin 11), FHL5 (STXBP2/Munc18-2), GS2 (RAB27A), HPS2, XLP1, XLP2, BLOC1S6,CD27, ITK, LYST, MAGT1 (XMEN), SLC7A7, and XIAP(BIRC4). Reactive (secondary/acquired) disease is triggered 
by infections, malignancies, rheumatologic disorders, immune deficiency syndromes, drugs, and metabolic diseases. ${ }^{1}$ In our patient, perforin (PRF1), syntaxin-11 (STX11), and Munc13-4 (UNC13D) gene mutation analyses were negative. Analysis for the other gene mutations was not investigated because of technical impossibility. In this case the diagnosis was based on the presence of 5 of 8 diagnostic criteria (fever, pancytopenia, splenomegaly, hypofibrinogenemia, hyperferritinemia, and hemophagocytosis in bone marrow). ${ }^{1,2}$

Metabolic diseases such as lysinuric protein intolerance, methylmalonic acidemia, propionic acidemia (PA), galactosemia, Gaucher disease, multiple sulfatase deficiencies, Pearson syndrome, galactosialidosis, and biotinidase deficiency were reported as underlying causes of HLH in the literature. ${ }^{3,4}$ There are no reports of greater frequency during the first year of life, when all causes of hemophagocytic lymphohistiocytosis are analyzed. In this case, both primary HLH gene mutation analysis and metabolic disease screening tests were performed simultaneously because congenital metabolic diseases may occur in the first year of life. Metabolic disease screening test results were compatible with PA in our patient.

Propionic acidemia is an extremely rare autosomal recessive intoxication type organic acidemia due to propionyl-CoA carboxylase activity defects. Patients with PA have abnormal accumulation of propionic acid, 3-hydroxypropionate, and methyl citrate. The clinical symptoms of the patients are mainly caused by these intermediate metabolites and manifest as feeding difficulties, vomiting, lethargy, coma, metabolic acidosis, pancytopenia, and hyperammonemia. ${ }^{6}$ The first presentation of our patient was lethargy, pallor, poor feeding, hyperammonemia, pancytopenia, and metabolic acidosis, similar to the clinical findings of PA. His elevated propionylcarnitine level was detected with tandem mass spectrometry and elevated 3-hydroxy- propionic acid and methylcitric acid were detected with urine organic acid analysis.

Secondary HLH associated with PA was reported in the current literature during metabolic crisis in children without infection. ${ }^{3,7,8}$ Gokce et al., ${ }^{3}$ and Kasapkara et al., ${ }^{7}$ reported three cases of secondary HLH associated with PA in the pediatric age group. Sulaiman et al., ${ }^{8}$ reported an unusual case of recurrent encephalopathy due to secondary HLH in a 16-year-old boy with PA. All of the cases mentioned above were previously diagnosed with propionic acidemia in an early period of their lives. Interestingly and differently, our patient was diagnosed with PA as the underlying cause after HLH diagnosis.

Propionic acidemia and other congenital metabolic diseases should be kept in mind as underlying causes of HLH occurring in the early period of life. Therefore, it is important to perform a detailed assessment of metabolic diseases when diagnosing HLH in infants. Tandem mass spectrometry with neonatal dried blood spot, especially, may be performed for diagnosis of metabolic disorders.

Written informed consent was obtained from the patient's parents.

\section{REFERENCES}

1. Janka GE. Familial and acquired hemophagocytic lymphohistiocytosis. Eur J Pediatr. 2007; 166(2):95-109.

2. Palazzi DL, McClain KL, Kaplan SL. Hemophagocytic syndrome in children: an important diagnostic consideration in fever of unknown origin. Clin Infect Dis. 2003; 36(3):306-12.

3. Gokce M, Unal O, Hismi B, Gumruk F, et al. Secondary hemophagocytosis in 3 patients with organic acidemia involving propionate metabolism. Pediatr Hematol Oncol. 2012; 29(1):92-8.

4. Kardas F, Patiroglu T, Unal E, Chiang SC, et al. Hemophagocytic syndrome in a 4-month-old infant with biotinidase deficiency. Pediatr Blood Cancer. 2012;59(1):191-3.

5. Henter JI, Horne A, Aricó M, Egeler RM, et al. HLH-2004: Diagnostic and therapeutic guidelines for hemophagocytic lymphohistiocytosis. Pediatr Blood Cancer. 2007; 48(2):124-31.

6. Fraser JL, Venditti CP. Methylmalonic and propionic acidemias: clinical management update. Curr Opin Pediatr. 2016; 28(6):682-93.

7. Kasapkara CS, Kangin M, Oflaz Ozmen B, Ozbek MN, et al. Secondary Hemophagocytosis in Propionic Acidemia. Iran J Pediatr. 2015; 25(3):e339.

8. Sulaiman RA, Shaheen MY, Al-Zaidan H, Al-Hassnan Z, et al. Hemophagocytic lymphohistiocytosis: A rare cause of recurrent encephalopathy. Intractable Rare Dis Res. 2016; 5(3):227-30. 